\title{
Testing of resveratrol microemulsion photostability and protective effect against UV induced oxidative stress
}

VAIDA JUŠKAITË*

KRISTINA RAMANAUSKIENĖ

VITALIS BRIEDIS

Lithuanian University of Health

Sciences, Department of Clinical

Pharmacy, 50161 Kaunas, Lithuania

Accepted February 26, 2017

Published online May 4, 2017

\begin{abstract}
Resveratrol is well known for its antioxidant activity and susceptibility to ultraviolet radiation. Development of formulations providing improved stability and relevant drug delivery of resveratrol is still a challenging task. The aim of this study was to determine protective characteristics of formulated microemulsions by evaluating photoisomerization of resveratrol and to investigate the effects of resveratrol on human keratinocyte cells under oxidative stress caused by ultraviolet radiation. Incorporation of resveratrol into microemulsions resulted in increased photostability of active compounds and the results demonstrated that photodegradation of resveratrol was significantly delayed. Results of biopharmaceutical evaluation in vitro demonstrated that up to $60 \%$ of resveratrol was released from microemulsions within 6 hours under a constant release rate profile. In vivo biological testing confirmed the ability of resveratrol to protect cells from oxidative stress and to increase cell viability. It was concluded that microemulsions might be considered in the development of UV light sensitive compounds.
\end{abstract}

Keywords: microemulsion, resveratrol, photostability, HaCaT cells, UVB, oxidative stress

Resveratrol (3,5,4'-trihydroxystilbene, RES) is a naturally occurring polyphenolic compound, which protects cells from oxidative stress (1). Due to its high antioxidant activity, resveratrol is considered to have a high potential to be applied in therapy and prophylaxis. Interest in this phytoalexin has increased over recent years and its protective effects in treatment of skin diseases, such as skin aging or skin cancer, have been confirmed (2). Research data demonstrated a high superoxide, hydroxyl, and other radical scavenging ability of resveratrol and its ability to suppress production of reactive oxygen species (ROS) (3).

Resveratrol ability to penetrate into skin is limited, thus the development of efficient carriers is a possible approach to reaching appropriate active compound concentrations in skin layers. Besides, the delivery system should guarantee adequate stability of resveratrol

\footnotetext{
* Correspondence; e-mail: vaida.juskaite@lsmuni.lt
} 
since high compound photosensivity to visible light and UV radiation has been determined. UV radiation and temperature induce rapid transformation of trans-resveratrol into the cis-isomeric form, resulting in decreased biological activity (4). The trans-isoform of resveratrol should be protected until its delivery to the site of action in skin tissues.

Physically and chemically stable nanoformulations are thought to provide active substances with efficient protection from external factors. Application of nanostructured formulations could help overcome the poor solubility of resveratrol and its limited penetration into the skin $(5,6)$. Microemulsions (MEs) are known for their ability to incorporate high amounts of lipophilic and hydrophilic active compounds and provide a skin penetration enhancing effect (7). Ease of formulation and thermodynamic stability are other important advantages of MEs that make them superior to other colloidal systems, e.g. nanoparticles, niosomes or liposomes (8). Consequently, it is useful to apply ME for incorporation of photolabile compounds (e.g. resveratrol) and suitability of the ME carrier has to be confirmed by stability testing and biopharmaceutical characterization of the formulation.

The aim of this study was to evaluate the ability of formulated MEs to protect resveratrol from photoisomerization and to assess their quality after UVB exposure. Also, ME irritation potential was evaluated by testing on a human keratinocyte (HaCaT) cell line. Non-toxic concentrations of MEs were determined by evaluating cell viability. Protective and antioxidant effects of resveratrol on human skin cells under normal conditions and under oxidative stress, caused by UVB radiation, were investigated.

\section{EXPERIMENTAL}

\section{Materials}

Analytical grade trans-resveratrol and chromatography grade acetonitrile and acetic acid were obtained from Sigma-Aldrich Chemie GmbH (Germany). Resveratrol was supplied by Naturex (France). MEs were formulated using PEG-8 caprylic/capric glycerides as surfactants and polyglyceryl-6-isostearate as cosurfactants, both supplied by Gattefosse (France). Ethyl oleate was used as an oily phase in MEs and was purchased from Alfa Aesar GmbH \& Co KG (Germany). Ultrapure water was produced using a water purification system Millipore Simplicity ${ }^{\mathrm{TM}}$ (USA). Dimethyl sulfoxide (DMSO) and $96 \%$ ethanol were used as solvents for resveratrol and were obtained from Sigma-Aldrich Chemie $\mathrm{GmbH}$ (Germany) and AB Vilniaus degtinė (Lithuania), respectively. Polyethylene glycol (PEG 400) was purchased from Carl Roth GmbH+Co KG (Germany). Trypsin, Dulbecco's modified Eagle's medium (DMEM), penicillin/streptomycin solution, fetal bovine serum (FBS), Hank's balanced salt solution (HBSS), and 3-(4,5-dimethylthiazol-2-yl)-2,5-diphenyltetrazolium bromide (MTT) were obtained from Life technologies (USA).

\section{Quantitative determination of trans-resveratrol}

Resveratrol was determined in all tested samples by validated high-performance liquid chromatography (HPLC) using an Agilent 1260 Infinity capillary LC system (Agilent Technologies Inc., USA) with a diode array detector. The Zorbax C18 column $(150 \times 0.5 \mathrm{~mm}$, $5 \mu \mathrm{m})$ was used for separation of trans- and cis-resveratrol. Separation was performed 
under isocratic conditions using a $0.5 \%$ aqueous solution of acetic acid (solvents A) and acetonitrile (solvent B) in a ratio 75:25 $(\mathrm{V} / \mathrm{V})$ as mobile phase. Eluent flow rate was $10 \mu \mathrm{L}$ $\mathrm{min}^{-1}$. UV detection was performed at a $306 \mathrm{~nm}$ wavelength. Column temperature was maintained at $25^{\circ} \mathrm{C}$.

\section{Formulation of microemulsions}

Composition of MEs was chosen considering our previous results of composition optimization, characterization, stability and skin penetration studies, and contained $57 \%$ of the surfactant and co-surfactant mixture, $38 \%$ of aqueous phase, and $5 \%$ of oily phase (9). Skin penetration studies demonstrated that highest quantities of resveratrol in the skin were determined when $2 \%$ resveratrol ME (ME-RES) with surfactant and co-surfactant mass ratio 5:1 was applied. Physical properties of ME-RES were defined by evaluating droplet size distribution and the mean particle size.

MEs with and without RES were formulated using the oil titration method at room temperature. A mixture of surfactant and co-surfactant $(5: 1, \mathrm{~m} / \mathrm{m})$ was added to water under mixing at $1250 \mathrm{rpm}$ for 10 minutes using a magnetic stirrer IKAMAG ${ }^{\circledR}$ C-MAG HS7 (IKA-Werke GmbH \& Co.KG, Germany). Ethyl oleate was added dropwise to the mixture under vigorous stirring until transparent and a clear formulation was obtained. ME-RES was formulated by dissolving RES in the oily phase and surfactant to produce a $2 \%$ concentration of active substance in the finished product (9). ME and ME-RES formation was confirmed visually. MEs were allowed to equilibrate for 24 hours keeping formulations at ambient temperature.

\section{Characterization of microemulsions}

The physical quality of MEs was evaluated by determining the mean droplet size and polydispersity index (PDI) of MEs using a Zetasizer Nano ZS particle size analyzer (UK). PDI was measured to characterize the droplet size distribution. All formulations were analyzed at $25^{\circ} \mathrm{C} 24 \mathrm{~h}$ after their production and measurements were performed in triplicate.

\section{Evaluation of resveratrol photostability}

Photostability studies were performed to determine the effect of UVB on the trans-RES to cis-RES transformation. Transformation process was evaluated by comparing RES photostability in a $2 \%$ ME-RES and $2 \%$ ethanolic resveratrol solution (RES-control). Stability of formulated MEs was evaluated by irradiating ME without resveratrol (ME-control). Irradiation of samples was performed in Petri dishes placed at a $20 \mathrm{~cm}$ distance from a MRL-58 Multiple-Ray UV lamp (Ultra-Violet Products Ltd, UK) and were exposed to the light source $<1 \mathrm{~mW} \mathrm{~cm}^{-2}$ for 4 hours under ambient conditions $(4,10)$. Irradiance was determined using a UVX radiometer (Ultra-Violet Products Ltd, UK). Aliquots were taken from $2 \%$ ME-RES and RES-control at 15, 30, 60, 120, and $240 \mathrm{~min}$, diluted with ethanol, and trans- and cis-RES were determined by HPLC. Physical stability of ME-RES and ME-control was evaluated by measuring the droplet size and PDI applying the dynamic light scattering technique after UBV irradiation for 120 and $240 \mathrm{~min}$. 


\section{In vitro release studies}

RES release from MEs was studied by measuring diffusion from a dialysis bag (5). ME-RES samples $(1.00 \pm 0.02 \mathrm{~g})$ were placed into the pre-swelled dialysis bag (Sigma-Aldrich Chemie $\mathrm{GmbH}$, Germany). The ends of the bag were fixed with clamps and immersed into $500 \mathrm{~mL}$ of dialysis medium. A mixture of purified water and PEG 400 (50:50, $\mathrm{m} / \mathrm{m}$ ) was used as acceptor medium and was stirred using a hotplate magnetic stirrer IKAMAG $^{\circledR}$ C-MAG HS7 (IKA-Werke GmbH \& Co.KG, Germany). Medium temperature was kept at $32 \pm 0.1{ }^{\circ} \mathrm{C}$. Sink conditions were maintained throughout release studies. $1 \mathrm{~mL}$ of acceptor medium was removed at time points of $0.5,1,2,4,6$ hours and replaced by the same volume of fresh dissolution medium. Withdrawn samples were filtered through 0.20 $\mu \mathrm{m}$ nylon membrane filters and analyzed by the HPLC method.

\section{Cell lines and cell culture}

The immortalized human keratynocyte cell line, HaCaT cells, was obtained from Cell Lines Service $\mathrm{GmbH}$ (Germany). The cells were seeded and grown in culture flasks with DMEM containing $10 \%$ fetal bovine serum (FBS) and $100 \mathrm{U} \mathrm{mL}^{-1}$ penicillin and $100 \mu \mathrm{g}$ $\mathrm{mL}^{-1}$ streptomycin. HaCaT cells were cultured at $37^{\circ} \mathrm{C}$ under humidified conditions with $5 \% \mathrm{CO}_{2}$. The cells were sub-cultured after reaching about $80 \%$ confluence and the medium was changed two or three times per week.

\section{Measurement of cell viability}

Cell viability was assessed by the MTT method (11). The cells were seeded into 96-well plates at a density of 20,000 cells/well and incubated for $24 \mathrm{~h}$. After incubation, cells were treated with different sample solutions: a) ME without added resveratrol (ME-control), b) ME-RES, and c) solution of resveratrol in DMSO (RES-control). All the prepared samples were diluted using Hank's balanced salt solution (HBSS). After $24 \mathrm{~h}$ of treatment with the preparations, DMEM medium was removed and cells were washed twice with HBSS (100 $\mu \mathrm{L} /$ well). After washing, $180 \mu \mathrm{L} /$ well HBSS and $20 \mu \mathrm{L} /$ well MTT (final concentration, $5 \mathrm{mg} /$ $\mathrm{mL}$ ) dye were added to each well, and cells were incubated for $2 \mathrm{~h}$ at $37^{\circ} \mathrm{C}$ under humidified conditions with $5 \% \mathrm{CO}_{2}$. After that, the dye was removed and DMSO $(100 \mu \mathrm{L} /$ well $)$ was added in order to dissolve the purple crystals of formazan formed. The plate with solvent was kept in the dark for $15 \mathrm{~min}$. Absorption was measured spectrophotometrically at $550 \mathrm{~nm}$ and $620 \mathrm{~nm}$ wavelengths using a microplate spectrophotometer (Thermo Scientific Multiscan FC, USA). Results of cellular viability were expressed as the percentage of untreated control cells. All experiments were repeated three times at least.

\section{Effect of formulations on cell damage caused by UVB}

Different doses of UVB irradiation $\left(10,25,50,75\right.$ and $\left.100 \mathrm{~mJ} \mathrm{~cm}{ }^{-2}\right)$ were used to cause cell damage and to evaluate the protective effect of tested formulations (ME-control, MERES, RES-control). The cells were seeded into 96-well plates (20,000 cells/well) and incubated for $24 \mathrm{~h}$. After incubation, HaCaT cells were treated with different doses of UVB irradiation to determine the dose-cell viability relationship. Later on, the possible protective effect of tested formulations on the irradiated cells was assessed by testing cell viability 
after UVB irradiation. Tested formulations were added to the HaCaT cells cultivated in 96-well plates $2 \mathrm{~h}$ prior to UVB irradiation (12). Before UVB exposure, the medium was removed and cells were washed once with HBSS. The cells were irradiated with UVB using a UV lamp (UVP, MRL-58 Multiple-Ray Lamp, USA) after HBSS thin layer addition. Irradiation dose was calculated using a UVX Radiometer (UVP, USA). HBSS was removed after irradiation. Fresh medium was added to each well and cells were incubated for $24 \mathrm{~h}$. Cell viability was determined by the MTT method as described above. Untreated cells and UVB irradiated cells without test formulations added were used as controls.

\section{Statistical analysis}

All tests were repeated three times at least. The results were expressed as means \pm SD. Statistically significant difference was determined when $p<0.05$. Student's $t$-test was used to evaluate significant differences in test results. Statistical analysis of experimental data was performed using the Microsoft Office Excel and 2013 SPSS software (version 19.0).

\section{RESULTS AND DISCUSSION}

Published research data demonstrated limited stability of RES under irradiation with UV light, which could cause photodegradation of RES (13). Microemulsions (MEs) were formulated to provide a protective effect on RES and minimize possible isomerization. In order to evaluate the protective effect of formulated ME on the photostability of RES under UVB exposure, the stability of trans-RES in RES-ME and ethanolic solution of RES (REScontrol) was tested. The concentration of trans-RES in tested formulations was $2 \%$.

Concentration of trans-RES decreased both in ME and in ethanolic solution under UVB exposure (Fig. 1). Results of photodegradation studies showed a decrease of trans-RES

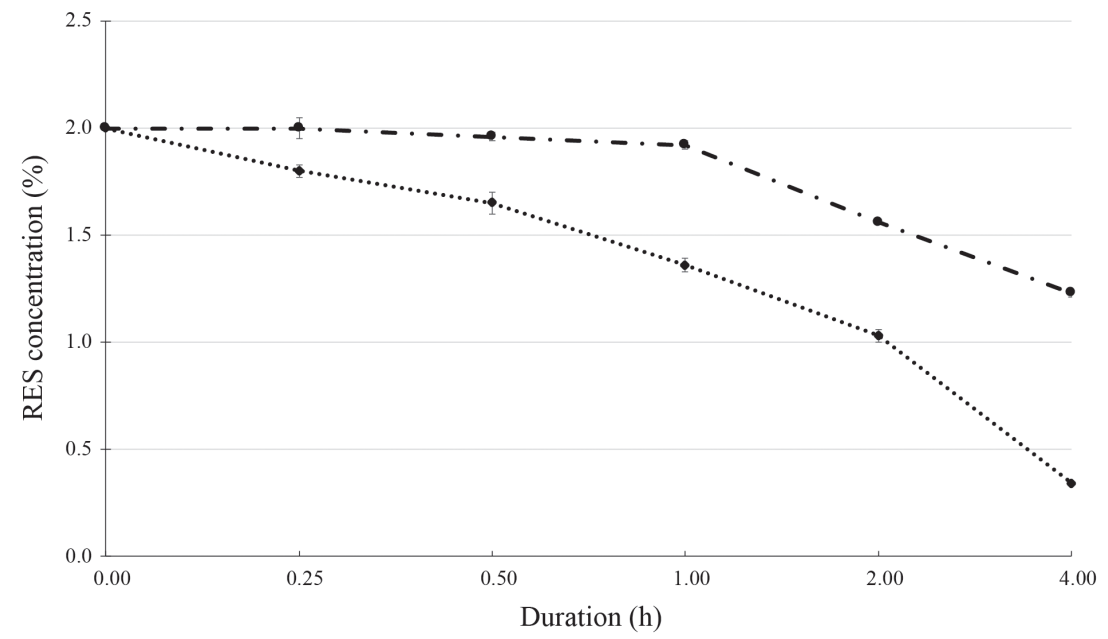

Fig. 1. Stability of $2 \%$ trans-resveratrol in microemulsion $(-\bullet-)$ and ethanolic solution (....) under UV irradiation. 
by $83.7 \%$ in ethanolic solution in $4 \mathrm{~h}$. Trans-RES concentration decreased by $39.1 \%$ after 4 $\mathrm{h}$ of UVB exposure when ME-RES formulation was irradiated. The results demonstrated that inclusion of RES in ME decreased photoisomerization of RES while concentration of trans-RES was approx. 3.2 fold higher. ME provided efficient protection to RES for $1 \mathrm{~h}$ under UVB irradiation. Concentration of trans-RES in RES-control was gradually decreasing over all the $4 \mathrm{~h}$ of exposure to UVB. Decrease of trans-RES was determined in samples collected at 2-h and 4-h time points. The results demonstrated the ability of MEs to provide increased physical stability of RES under UVB irradiation compared to ethanolic solution. These results could be related to physicochemical characteristics of the active substance since lipophilic RES predominantly accumulated in droplets' interfacial layer and was less accessible to UVB radiation. Similar observations were reported when application of nanostructured formulations (nanocapsules, nanospheres, liposomes, nanostructured lipid carriers) decreased RES photodegradation significantly $(4,10)$.

The effect of UVB irradiation on ME particle size characteristics was evaluated by measuring the mean droplet size and PDI before and after UVB exposure. Smaller droplets of ME-control were determined compared to droplet size when ME contained RES (Table I). The increase in droplet size could be due to incorporation of RES as a fraction of surfactant and co-surfactant should participate in solubilization of RES molecules. Decreased availability of surfactant and co-surfactant molecules resulted in a decrease of interfacial film surface area and a proportional increase in droplet size. However, the size of ME-RES was in an acceptable ME droplet size range. Similar effects of active substances on droplet size were reported previously when active substances were incorporated into colloidal systems, e.g. microemulsions (14), liposomes (10), nanosuspensions (15). The possibility of RES accumulation in the interfacial layer of nanostructure systems has been discussed earlier (6). Incorporation of RES into MEs did not affect PDI values of the system compared to ME-control and the narrow droplet size distribution indicated homogeneity of formulations.

The results demonstrated increased mean droplet size with increasing exposure to UVB. Mean droplet size in ME-RES was 1.7 fold bigger compared to the mean droplet size in ME-control before UVB irradiation ( $0 \mathrm{~h}$ ). After $4 \mathrm{~h}$ of UVB exposure, the mean droplet size increased by $37.2 \%$ in ME-RES and by $65.41 \%$ in ME-control compared to the mean droplet size before UVB irradiation. This indicates that UV irradiation can induce changes in the chemical composition of the interfacial film and lower the surface activity of possibly formed compounds. The presence of RES in the ME interfacial film increases its resistance against the damaging UV effect. Study results demonstrated a decrease in PDI of ME-control (from 0.286 to 0.242 ) and a single peak droplet size distribution pattern. Expo-

Table I. Characteristics of MES-RES and MEs before and after UVB irradiation

\begin{tabular}{ccccc}
\hline \multirow{2}{*}{$\begin{array}{c}\text { Duration of UVB } \\
\text { irradiation (h) }\end{array}$} & \multicolumn{2}{c}{ ME-RES } & \multicolumn{2}{c}{ ME-control } \\
\cline { 2 - 5 } & Droplet size (nm) & PDI & Droplet size (nm) & PDI \\
\hline 0 & $112.5 \pm 1.3$ & $0.263 \pm 0.008$ & $66.3 \pm 0.2$ & $0.286 \pm 0.005$ \\
2 & $132.4 \pm 2.6$ & $0.301 \pm 0.024$ & - & - \\
4 & $154.4 \pm 2.0$ & $0.316 \pm 0.041$ & $109.7 \pm 0.7$ & $0.242 \pm 0.008$ \\
\hline
\end{tabular}




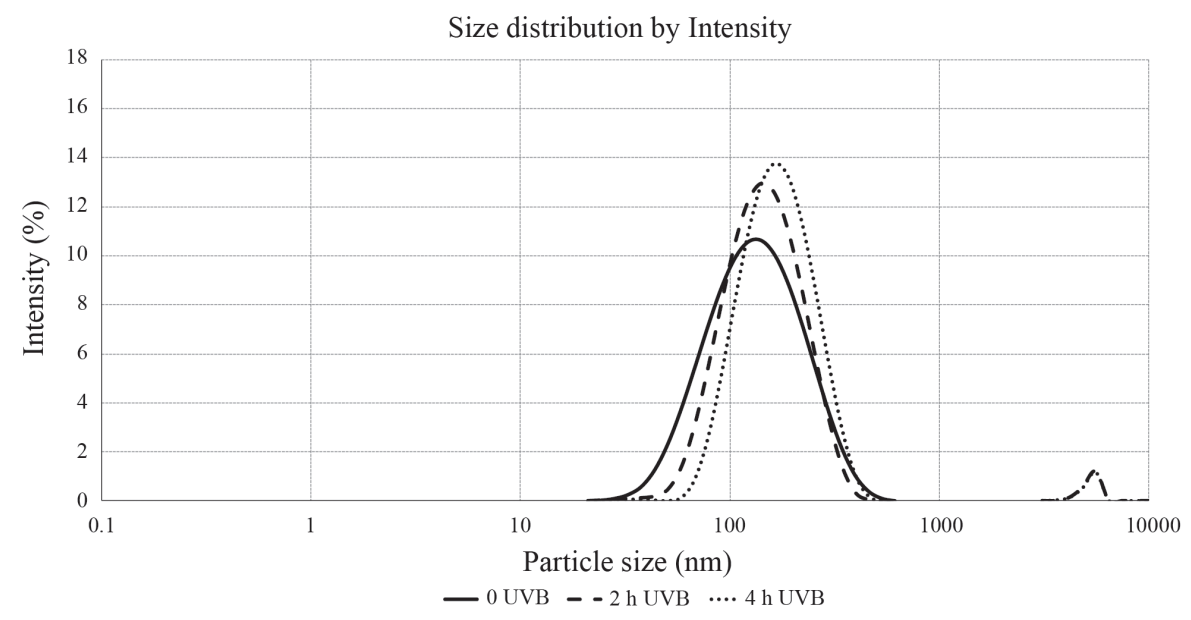

Fig. 2. Effect of UVB irradiation on droplet size distribution in ME-RES.

sure of MEs to UVB resulted in increased PDI and a two-peak droplet size distribution in ME-RES, indicating the appearance of bigger droplets (Fig. 2).

Droplet size distribution was investigated to evaluate the physical stability of the carrier before and after UVB exposure. Study results demonstrated that $24 \mathrm{~h}$ after formulation the droplet size in ME-RES ranged from 28.21 to $531.2 \mathrm{~nm}$. Changes of ME-RES droplet size were observed after 2 and $4 \mathrm{~h}$ of UVB irradiation. After $2 \mathrm{~h}$ of UVB exposure, droplet sizes were in the ranges of $28.21-396.1 \mathrm{~nm}$ and $4145-5560 \mathrm{~nm}$. Droplet size measurements demonstrated the formation of bigger droplets (32.67-458.7 $\mathrm{nm}$ and $4145-5560 \mathrm{~nm}$ ) after $4 \mathrm{~h}$ of UVB radiation, but the droplet mean size of the second peak did not change. The results of intensity measurements demonstrated that the predominating particle fraction in the main peak remained the same as before UV irradiation (Fig. 2); however, the appearance of a bigger particle peak was identified. The second peak indicates formation of bigger particles, which could represent the products of possible coalescence of ME droplets or their agglomeration. The applied photon correlation spectroscopy method did not allow reliable differentiation of droplets and agglomerates. However, the applied method confirmed the presence of bigger particles and pointed to the destabilizing effect of UV irradiation on the MEs formulations tested. Evaluation of ME components' individual susceptibility to UV light could provide information for a better understanding of processes that potentially induce changes in MEs. Stabilization of MEs against the UV effect could be achieved either by including antioxidants or by using surfactants possessing antioxidant properties for interfacial film formation (16).

The ability of the formulated system to release active substances is considered to be an important criterion in product characterization and quality evaluation studies. Determination of the RES release profile provided additional understanding of the distribution of RES in ME. RES is considered to have limited solubility in water and it required a modified acceptor medium compared to water-soluble drugs. As regards solubility characteristics, PEG 400 was added to acceptor medium to maintain sink conditions throughout the testing pro- 
cedure. Results of biopharmaceutical evaluation revealed that RES was released at a constant speed and the total amount of released RES reached $60.73 \%$ after $6 \mathrm{~h}$. However, the delay of RES appearance in acceptor medium in the initial phase of the experiment could be attributed to the limited diffusion process across the semipermeable membrane and release of RES from ME droplets (5). Results of RES release evaluation demonstrated a constant release rate over the $6 \mathrm{~h}$ of testing. A zero order kinetic model was used to describe release kinetics of the drug from the formulated system $\left(R^{2}=0.992\right)$. Results of drug release studies confirmed the suitability of the carrier to incorporate and release RES, and ME-RES formulation was considered acceptable for further biological testing.

Effects of ME excipients in their formulation on manifestation of RES biological activity should be confirmed by biological testing on appropriate cell models. Biological evaluation of the produced formulations was done applying the human keratinocyte (HaCaT) cellular model. Different concentrations of ME-control were used to evaluate its potential cytotoxicity. No statistically significant difference was established $(p>0.05)$ between the viability of control group cells (untreated cells) and the viability of cells exposed to MEcontrol at concentrations of $0.01,0.05,0.1,0.15$ and $0.2 \%$. Exposure of keratinocytes to 0.25 $\%$ or higher concentrations of ME-control resulted in a statistically significant difference $(p<0.05)$ of cell viability compared to the control since the viability of keratinocytes was less than $90 \%$. Considering these results, the $0.15 \%$ concentration of ME was chosen for further studies.

Protective effects of formulated systems against oxidative stress damage were evaluated using UVB as inducer of ROS generation. UVB is a major factor in the development of skin diseases, particularly skin cancer $(3,12)$. The viability of UVB affected HaCaT cells was evaluated using the MTT method. Five different UVB doses $(10,25,50,75$, and $100 \mathrm{~mJ}$ $\mathrm{cm}^{-2}$ ) were applied to evaluate the effects of UVB irradiation on cell viability (Fig. 3). Study results demonstrated that UVB exposure caused a statistically significant $p<0.05$ ) decrease of cell viability. Viability evaluation showed that cell viability depends on the UVB dose, since its increase resulted in decreased numbers of viable cells. The results in Figure 3 show a two-fold decrease of $\mathrm{HaCaT}$ viable cells after exposure to a $50 \mathrm{~mJ} \mathrm{~cm}^{-2}$ dose compared to non-irradiated cells. Maximum decrease (75.32\%) was observed after $100 \mathrm{~mJ} \mathrm{~cm}{ }^{-2}$ UVB exposure.

Results of the experiment indicated decreased cell survival after UVB irradiation, thus different formulations were tested to evaluate their protective potential for $\mathrm{HaCaT}$ cells. Keratinocytes were pretreated with tested formulations (ME-control, ME-RES, REScontrol) for 2 hours prior irradiation with UVB and the protective effect of RES was evaluated. RES concentration in cell containing wells was $131 \mu \mathrm{M}$.

Study results demonstrated that treatment of cells with ME-control and following UVB irradiation had no effect on the viability of cells in the tested irradiation range from $10 \mathrm{~mJ} \mathrm{~cm}^{-2}$ to $100 \mathrm{~mJ} \mathrm{~cm}^{-2}$ compared to UVB irradiated cells (Fig. 3). Pretreatment of cells with RES, applied in ME (ME-RES) and in solution (RES-control) before UVB exposure, resulted in increased $\mathrm{HaCaT}$ cell viability. No statistically significant difference was established $(p>0.05)$ between the viability of cells subjected to UVB (UVB-control) and RES pretreated cells when radiation doses of 10 and $25 \mathrm{~mJ} \mathrm{~cm}^{-2}$ were applied. Increased RES photoprotection was identified at higher doses of UVB $\left(>50 \mathrm{~mJ} \mathrm{~cm}^{-2}\right)$ and cell viability differed significantly $(p<0.05)$ compared to UVB irradiated control cells. Protective effect of ME-RES was determined when keratinocytes were irradiated with UVB doses ranging 


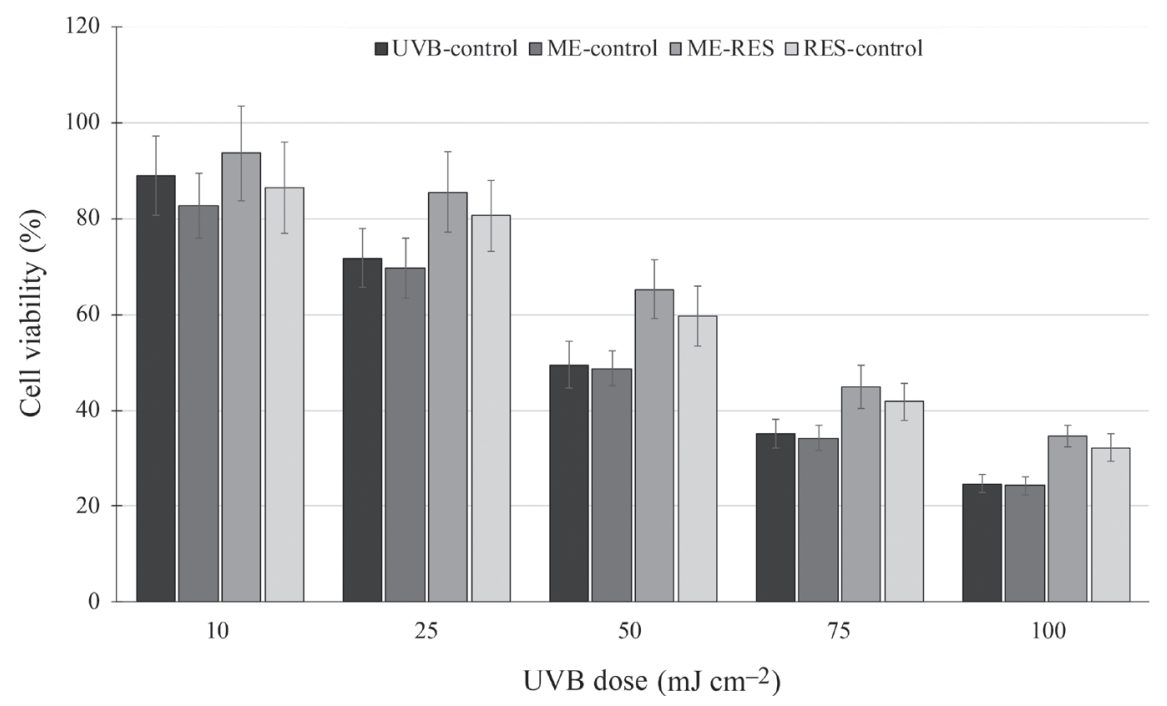

Fig. 3. Effect of the presence of resveratrol in applied formulations and of UVB dose on HaCaT cell viability.

from 50 to $100 \mathrm{~mJ} \mathrm{~cm}^{-2}$ cell and cell viability increased by $27.8-40.1 \%$ compared to UVBcontrol. A similar protective effect on cell viability was established when results obtained after ME-RES application were compared to ME-control. Application of RES in ME resulted in higher cell viability than RES-control even though differences were not statistically significant in the UVB irradiation ranges tested. The above findings provide a rationale for using MEs in the delivery of RES considering increased RES stability in ME and delayed release pattern.

These studies also confirmed the photoprotective effect of RES and its ability to reduce UVB radiation caused cell death. Protective effects of RES on stressed keratinocytes could be associated with its antioxidant activity. Epidermis has a specific binding site for RES, thus topical application of RES is considered an effective approach in prevention and treatment of skin diseases (1). The results of this study demonstrate the ability of formulated ME to function as an efficient RES delivery system for transporting it into epidermal cells, and thus confirms the significant protective effect of RES against oxidative stress.

\section{CONCLUSIONS}

MEs are spontaneously forming disperse systems and were formulated as potential carriers for delivery of RES into the skin. Physical stability, drug release and biological studies were performed to determine the suitability of the formulated nanocarrier system. The results demonstrated that MEs could protect RES from photoisomerization and ensure the stability of RES for up to 1 hour under UVB irradiation. Drug release testing revealed diffusion and gradual release of RES from ME. The effects of RES on skin cells confirmed the potential use of this antioxidant as a protective agent to reduce ROS generation and enhance 
cell viability when the cells were subjected to oxidative stress. All these findings indicate that the formulated ME-RES could be considered a drug delivery system for RES topical application although the increase in droplet size of MEs under UV radiation was determined.

\section{REFERENCES}

1. M. Ndiaye, C. Philippe, H. Mukhtar and N. Ahmad, The grape antioxidant resveratrol for skin disorders: Promise, prospects, and challenges, Arch. Biochem. Biophys. 508 (2011) 164-170; DOI: 10.1016/j.abb.2010.12.030.

2. R. A. Baxter, Anti-aging properties of resveratrol: Review and report of a potent new antioxidant skin care formulation, J. Cosmetic Dermatol. 2008, 2-7; DOI: 10.1111/j.1473-2165.2008.00354.x.

3. S. S. Leonard, C. Xia, B. H. Jiang, B. Stinefelt, H. Klandorf, G. K. Harris and X. Shi, Resveratrol scavenges reactive oxygen species and effects radical-induced cellular responses, Biochem. Biophys. Res. Commun. 309 (2003) 1017-1026; DOI: 10.1016/j.bbrc.2003.08.105.

4. C. B. Detoni, G. D. Souto, A. L. M. Da Silva, A. R. Pohlmann and S. S. Guterres, Photostability and skin penetration of different E-resveratrol-loaded supramolecular structures, Photoc. Photobiol. 2012, 913-921; DOI: 10.1111/j.1751-1097.2012.01147.x.

5. K. Teskač and J. Kristl, The evidence for solid lipid nanoparticles mediated cell uptake of resveratrol, Int. J. Pharm. 390 (2009) 61-69; DOI: 10.1016/j.ijpharm.2009.10.011.

6. C. Caddeo, M. Manconi, A. M. Fadda, F. Lai, S. Lampis, O. Diez-Sales and C. Sinico, Nanocarriers for antioxidant resveratrol: Formulation approach, vesicle self-assembly and stability evaluation, Coll. Surf. B. Biointerfaces 111 (2013) 327-332; DOI: 10.1016/j.colsurfb.2013.06.016.

7. H. Y. Karasulu, Microemulsions as novel drug carriers: the formation, stability, applications and toxicity, Expert Opin. Drug Deliv. 5 (2008) 119-135; DOI: 10.1517/17425247.5.1.119.

8. T. Wan, T. Xu, J. Pan, M. Qin, W. Pan, G. Zhang, Z. Wu, C. Wu and Y. Xu, Microemulsion based gel for topical dermal delivery of pseudolaric acid B: In vitro and in vivo evaluation, Int. J. Pharm. 493 (2015) 111-120; DOI: 10.1016/j.ijpharm.2015.07.058.

9. V. Juškaite, K. Ramanauskienė and V. Briedis, Design and formulation of optimized microemulsions for dermal delivery of resveratrol, eCAM 2015, 1-10; DOI: 10.1155/2015/540916.

10. S. Sapino, M. E. Carlotti, G. Caron, E. Ugazio and R. Cavalli, In silico design, photostability and biological properties of the complex resveratrol/hydroxypropyl- $\beta$-cyclodextrin, J. Incl. Phenom. Macrocycl. Chem. 63 (2009) 171-180; DOI: 10.1007/s10847-008-9504-7.

11. K. Ramanauskiene, A. Stelmakiene and D. Majiene, Assessment of Lemon Balm (Melissa officinalis L.) Hydrogels: Quality and Bioactivity in Skin Cells, eCAM 2015, 1-7; DOI: 10.1155/2015/635975.

12. N. Vitale, A. Kisslinger, S. Paladino, C. Procaccini, G. Matarese, G. M. Pierantoni, F. P. Mancini and D. Tramontano, Resveratrol couples apoptosis with autophagy in UVB-irradiated HaCaT cells, PLoS One. 8 (2013) e80728; DOI: 10.1371/journal.pone.0080728.

13. A. Francioso, A. Boffi, C. Villani, L. Manzi, M. D’Erme, A. Macone and L. Mosca, Isolation and identification of 2,4,6-trihydroxyphenanthrene as a byproduct of trans-resveratrol photochemical isomerization and electrocyclization, J. Org. Chem. 79 (2014) 9381-9384; DOI: 10.1021/jo501405m.

14. P. Sasivimolphan, V. Lipipun, G. Ritthidej, K. Chitphet, Y. Yoshida, T. Daikoku, B. Sritularak, K. Likhitwitayawuid, P. Pramyothin, M. Hattori and K. Shiraki, Microemulsion-based oxyresveratrol for topical treatment of herpes simplex virus (HSV) infection: Physicochemical properties and efficacy in cutaneous HSV-1 infection in mice, AAPS PharmSciTech. 13 (2012) 1266-1275; DOI: 10.1208/s12249-012-9828-x.

15. J. Hao, Y. Gao, J. Zhao, J. Zhang, Q. Li, Z. Zhao and Y. Liu, Preparation and optimization of resveratrol nanosuspensions by antisolvent precipitation using Box-Behnken design, AAPS PharmSciTech. 16 (2015) 118-128; DOI: 10.1208/s12249-014-0211-y.

16. H. Chen and Q. Zhong, Thermal and UV stability of $\beta$-carotene dissolved in peppermint oil microemulsified by sunflower lecithin and Tween 20 blend, Food Chem. 174 (2015) 630-636; DOI: 10.1016/j.foodchem.2014.11.116. 